\title{
HODGE DECOMPOSITIONS AND DOLBEAULT COMPLEXES ON NORMAL SURFACES
}

\author{
JEFFREY FOX AND PETER HASKELL
}

\begin{abstract}
Give the smooth subset of a normal singular complex projective surface the metric induced from the ambient projective space. The $L^{2}$ cohomology of this incomplete manifold is isomorphic to the surface's intersection cohomology, which has a natural Hodge decomposition. This paper identifies Dolbeault complexes whose $\bar{\partial}$-closed and $\bar{\partial}$-coclosed forms represent the classes of pure type in the corresponding Hodge decomposition of $L^{2}$ cohomology.
\end{abstract}

\section{INTRODUCTION}

Among the early motivations for the study of both the $L^{2}$ cohomology of smooth subsets of certain singular spaces and the intersection (co)homology of certain singular spaces was the desire to extend to classes of singular spaces the properties of the (co)homology of smooth oriented manifolds. [C2, C3, C4, GM1, GM2] The observation that the smooth part of a singular algebraic variety sometimes carries a natural Kähler metric for which the $L^{2}$ cohomology is isomorphic to the intersection cohomology of the variety stimulated interest in finding pure Hodge decompositions of $L^{2}$ cohomology and intersection cohomology $[\mathrm{C} 1, \mathrm{C} 2, \mathrm{CGM}]$. Using methods not directly related to $L^{2}$ cohomology, Saito $[\mathrm{S}]$ has proven that the intersection cohomology of a singular variety has a pure Hodge decomposition.

The efforts to find a pure Hodge structure for $L^{2}$ cohomology and to identify $L^{2}$ cohomology with intersection cohomology have provided interesting and useful results both before and after Saito's discovery. The smooth part of a locally symmetric variety and of a variety with isolated singularities can be given a complete Kähler metric for which the $L^{2}$ cohomology is isomorphic to the intersection cohomology of the variety. [B, BCa, L, Sa1, Sa2, SaSt1, SaSt2, Z2, Z3]. The $L^{2}$ cohomology for the complete metric has a pure Hodge structure. For varieties with isolated singularities, the intersection cohomology has a "canonical" pure Hodge structure that agrees with the one arising in the $L^{2}$ cohomology associated with the above complete metric [Z1]. 1992.

Received by the editors June 5, 1990 and, in revised form, July 21, 1992 and November 23,

1991 Mathematics Subject Classification. Primary 58G05, 14C30, 58A14.

Key words and phrases. Normal surface, induced metric, $L^{2}$ Dolbeault cohomology, Hodge decomposition.

The first author was supported by the National Science Foundation under Grant No. DMS8903472. The second author was supported by the National Science Foundation under Grant No. DMS-8901436. 
The smooth part of any variety inherits an incomplete Kähler metric from the ambient projective space. If the variety is a curve, this induced metric is conical. The $L^{2}$ cohomology and the intersection cohomology of the curve are isomorphic to each other and to the analogous cohomology of the curve's desingularization, from which the $L^{2}$ and intersection cohomology receive a pure Hodge structure [C2, CGM]. Remark 2.2 describes the relationship between this Hodge structure and Dolbeault complexes on the smooth part of the singular curve. If the variety is a normal surface, the $L^{2}$ cohomology in the induced metric and the intersection cohomology are again isomorphic [HsP, N3]. In a more complicated manner they receive a pure Hodge structure from the cohomology of a desingularization of the variety [CGM].

The purpose of this paper is to describe Dolbeault complexes whose $\bar{\partial}$-closed and $\bar{\partial}$-coclosed forms provide the pure Hodge structure of [CGM] on the $L^{2}$ cohomology of normal surfaces with induced metrics. We also prove a version of the hard Lefschetz theorem for these " $\bar{\partial}$-harmonic" forms. The list of references makes clear our dependence on papers by many authors, but the direct foundation for our proofs comes from [N2] and [H2]. The former paper describes properties, including a pure $(p, q)$-decomposition and a hard Lefschetz theorem, of the forms in the intersection of kernel $(d)$ and kernel $(* d *)$. (Here $d$ represents the maximal extension of exterior differentiation.) These forms are the harmonic representatives of $L^{2}$ cohomology classes in all degrees except perhaps degree 2 , and their $(p, q)$ type provides the Hodge decomposition of [CGM] for $p+q \neq 2$. Using the analysis of the minimal Dolbeault complex of $(0, q)$-forms appearing in [H2], the present paper constructs Dolbeault complexes whose $\bar{\partial}$-closed and $\bar{\partial}$-coclosed forms are the harmonic forms described by [N2] in total degree $\neq 2$ and whose $\bar{\partial}$-closed and $\bar{\partial}$-coclosed forms of type $(p, q)$, for all $p$ and $q$, represent the $L^{2}$ cohomology classes of type $(p, q)$ described in [CGM]. These Dolbeault complexes are minimal for the $(0, q)$ complex; maximal for the $(2, q)$ complex; and based on domain $\left(d_{c} d_{c}^{*}+d_{c}^{*} d_{c}\right)$ for the $(1, q)$ complex. (Definition 1.3 defines $d_{c}$.)

\section{DEFINITIONS AND NOTATION}

Henceforth $X$ denotes a normal singular complex projective algebraic surface. The set of smooth points of $X$ is called $U$. We give $U$ the metric induced from the Fubini-Study metric on the ambient projective space. This makes $U$ an incomplete Kähler manifold.

Let $\pi: \tilde{X} \rightarrow X$ denote a desingularization of $X$. Let $\tilde{U}$ denote $\pi^{-1}(U)$. The restriction of $\pi$ realizes an isomorphism of the complex manifolds $\tilde{U}$ and $U$. Give the name $E$ to $\tilde{X} \backslash \tilde{U}$ and the name $\gamma$ to the pseudometric on $\tilde{X}$ that arises as the pullback under $\pi$ of the metric on $U$.

We require that $\pi: \tilde{X} \rightarrow X$ have the following properties. $E$ is a codimension one subvariety of $X$ with (at worst) simple normal crossings. For each smooth point of $E$, there are integers $m$ and $n, n \geq m \geq 1$, such that locally around the point on $E, \pi^{-1}(\operatorname{sing}(X))=\left(u^{m}\right)$ as a subvariety, and $\gamma$ is quasi-isometric to $d \varphi d \bar{\varphi}+d \psi d \bar{\psi}$. Here $\varphi(u, v)=u^{m}$ and $\psi(u, v)=u^{n} v$, and $u$ and $v$ are local coordinates on the complex manifold $\tilde{X}$. For each normal crossing of $E$, there are integers $m_{1}, n_{1}, m_{2}, n_{2}$ satisfying $n_{1} \geq m_{1} \geq 1$, $n_{2} \geq m_{2} \geq 1$, and $m_{1} n_{2}-m_{2} n_{1} \neq 0$. Locally around a crossing point on 
$E, \pi^{-1}(\operatorname{sing}(X))=\left(u^{m_{1}} v^{m_{2}}\right)$ as a subvariety, and $\gamma$ is quasi-isometric to $d \varphi d \bar{\varphi}+d \psi \bar{\psi}$. Here $\varphi(u, v)=u^{m_{1}} v^{m_{2}}$ and $\psi(u, v)=u^{n_{1}} v^{n_{2}}$, and again $u$ and $v$ are local coordinates on $\tilde{X}$. Normal surfaces always have desingularizations with these properties. (See [HsP] and [Pa, Theorem 1.4].)

Definition 1.1. Using the metric on $U$, we can define $\mathscr{L}^{i}(U)$, the Hilbert space of $L^{2} i$-forms on $U$, and $\mathscr{L}^{p, q}(U)$, the Hilbert space of $L^{2}$ forms of type $(p, q)$ on $U . \mathscr{L}^{i}(U)=\bigoplus_{p+q=i} \mathscr{L}^{p, q}(U)$, an orthogonal direct sum of Hilbert spaces.

Definition 1.2. Let $\underline{d}^{i}$ denote exterior differentiation defined on $\left\{w \in \mathscr{L}^{i}(U)\right.$ : $w$ is smooth and $\left.\underline{d}^{i} w \in \mathscr{L}^{i+1}(U)\right\}$. Let $d^{i}$ be the closure of $\underline{d}^{i}$, regarded as an unbounded operator $\mathscr{L}^{i}(U) \rightarrow \mathscr{L}^{i+1}(U)$.

Definition 1.3. Let $\underline{d}_{c}^{i}$ denote exterior differentiation defined on smooth compactly supported $i$-forms on $U\}$. Let $d_{c}^{i}$ denote the closure of $\underline{d}_{c}^{i}$, regarded as an unbounded operator $\mathscr{L}^{i}(U) \rightarrow \mathscr{L}^{i+1}(U)$.

Remark 1.4. The adjoint operators $\delta_{c}^{i+1}=\left(d^{i}\right)^{*}$ and $\delta^{i+1}=\left(d_{c}^{i}\right)^{*}$ are defined analogously using the differential operators $\underline{\delta}_{c}=-* \underline{d}_{c} *$ and $\underline{\delta}=-* \underline{d} *$.

Definition 1.5. Let $A^{i}(U)=\left\{w \in \mathscr{L}^{i}(U): w\right.$ is smooth and $\left.d^{i} w \in \mathscr{L}^{i+1}(U)\right\}$.

Definition 1.6. Let $A_{c}^{i}(U)=\left\{\right.$ smooth forms in domain $\left.\left(d_{c}^{i}\right)\right\}$.

Definition 1.7. Let $\underline{\partial}^{p, q}$ denote the Dolbeault operator defined on $\{w \in$ $\mathscr{L}^{p, q}(U): w$ is smooth and $\left.\underline{\partial}^{p, q} w \in \mathscr{L}^{p, q+1}(U)\right\}$. Let $\bar{\partial}^{p, q}$ be the closure of $\bar{\partial}^{p, q}$ regarded as an unbounded operator $\mathscr{L}^{p, q}(U) \rightarrow \mathscr{L}^{p, q+1}(U)$.

Definition 1.8. Let $\bar{\partial}_{c}^{p, q}$ denote the Dolbeault operator defined on ssmooth compactly supported $(p, q)$-forms on $\mathrm{U}\}$. Let $\bar{\partial}_{c}^{p, q}$ denote the closure of $\underline{\partial}_{c}^{p, q}$, regarded as an unbounded operator $\mathscr{L}^{p, q}(U) \rightarrow \mathscr{L}^{p, q+1}(U)$.

Remark 1.9. The adjoint operators $\left(\bar{\partial}^{p, q}\right)^{*}$ and $\left(\bar{\partial}_{c}^{p, q}\right)^{*}$ are defined analogously using $-* \underline{\bar{\partial}}_{c} *$ and $-* \underline{\bar{\partial}} *$.

Remark 1.10. The indexing of adjoint operators differs from that used in [N2]. We suppress indices from the notation for differential operators when the indices are clear from context.

Definition 1.11. Let $\mathscr{A}^{p, q}(U)=\left\{w \in \mathscr{L}^{p, q}(U): w\right.$ is smooth and $\bar{\partial} w \in$ $\left.\mathscr{L}^{p, q+1}(U)\right\}$.

Definition 1.12. Let $\mathscr{A}_{c}^{p, q}(U)=\left\{\right.$ smooth forms in domain $\left.\left(\bar{\partial}_{c}^{p, q}\right)\right\}$.

Definition 1.13. Using a nondegenerate Kähler metric on $\tilde{X}$ and its restriction to $\tilde{U}$, we can define Hilbert spaces $\mathscr{L}^{i}(\tilde{X}), \mathscr{L}^{p, q}(\tilde{X}), \mathscr{L}^{i}(\tilde{U})$, and $\mathscr{L}^{p, q}(\tilde{U})$ as in Definition 1.1.

Remark 1.14. Restriction induces isomorphisms

$$
\mathscr{L}^{i}(\tilde{X}) \cong \mathscr{L}^{i}(\tilde{U}) \text { and } \mathscr{L}^{p, q}(\tilde{X}) \cong \mathscr{L}^{p, q}(\tilde{U})
$$

Exterior differentiation $d$ and the Dolbeault operator $\bar{\partial}$ have unique closed extensions on $\tilde{X}$. 
Theorem 1.15 [HsP, N3]. The complex $\left(A^{i}(U), d^{i}\right)$ has finite-dimensional cohomology groups $H_{(2)}^{i}(U)$ isomorphic to the intersection cohomology groups of $X$.

Theorem 1.16 [N2, Theorem 1]. Let $\mathscr{H}^{i}=\operatorname{kernel}\left(d^{i}\right) \cap \operatorname{kernel}\left(\delta^{i}\right)$. Let $\mathscr{H}^{p, q}=$ $\mathscr{L}^{p, q}(U) \cap \mathscr{H}^{i}$ for $i=p+q$. Then

$$
\mathscr{H}^{i}=\bigoplus_{p+q=i} \mathscr{H}^{p, q} .
$$

Definition 1.17. Let $\hat{\mathscr{H}}^{i}=\operatorname{kernel}\left(d^{i}\right) \cap \operatorname{kernel}\left(\delta_{c}^{i}\right)$. Let $\hat{\mathscr{H}}^{p, q}=\mathscr{L}^{p, q}(U) \cap \hat{\mathscr{H}}^{i}$ for $i=p+q$.

Remark 1.18. Because $\delta_{c}^{i}=\left(d^{i-1}\right)^{*}, \hat{\mathscr{H}}^{i} \cong H_{(2)}^{i}(U)$. Note that $\hat{\mathscr{H}}^{i} \subset \mathscr{H}^{i}$ and $\hat{\mathscr{H}}^{p, q} \subset \mathscr{H}^{p, q}$ but that it does not necessarily follow that $\hat{\mathscr{H}}^{i}=\bigoplus_{p+q=i} \hat{\mathscr{H}}^{p, q}$.

Remark 1.19. On the compact manifold $\tilde{X}$ the set of smooth forms is exactly the set of smooth forms with compact support. The distinctions between $d$ and $d_{c}$, or between $\delta$ and $\delta_{c}$, that must be considered on $U$ do not arise on $\tilde{X}$. Indeed the Laplace operator $d \delta+\delta d$ defined on smooth forms on $\tilde{X}$ is essentially self-adjoint when viewed as an unbounded operator on $L^{2}$ forms on $\tilde{X}$. Harmonic forms on $\tilde{X}$ can be defined as $\operatorname{kernel}(d) \cap \operatorname{kernel}(\delta)$ or as $\operatorname{kernel}(\Delta)$. Both definitions lead to the same set of harmonic forms, which we will call $\mathscr{H}^{i}(\tilde{X})$. By elliptic regularity such harmonic forms are smooth even when the operators $d, \delta$, and $\Delta$ are interpreted as closed unbounded operators on Hilbert spaces of $L^{2}$ forms. $\mathscr{H}^{i}(\tilde{X})=\bigoplus_{p+q=i} \mathscr{H}^{p, q}(\tilde{X})$, where these summands may be defined as in Theorem 1.16 or as the set of $\bar{\partial}$-closed and $\bar{\partial}$-coclosed forms.

Definition 1.20. Let $\mathscr{D}^{1, q}(U)=\mathscr{L}^{1, q}(U) \cap$ domain $\left(d_{c} d_{c}^{*}+d_{c}^{*} d_{c}\right)$.

Remark 1.21. We are interested in the following subcomplex of $\left(\mathscr{L}^{1, q}(U)\right.$, $\left.\bar{\partial}^{1, q}\right)$.

$$
\mathscr{D}^{1,0}(U) \stackrel{\bar{\partial}^{1,0}}{\longrightarrow} \mathscr{D}^{1,1}(U) \stackrel{\bar{\partial}^{1,1}}{\longrightarrow} \mathscr{D}^{1,2}(U) .
$$

Here the operator $\bar{\partial}^{1, q}$ refers to the restriction of $\bar{\partial}^{1, q}: \mathscr{L}^{1, q}(U) \rightarrow \mathscr{L}^{1, q+1}(U)$ to the $\left\{w \in \mathscr{D}^{1, q}(U): \bar{\partial} w \in \mathscr{D}^{1, q+1}(U)\right\}$.

Remark 1.22. In what follows orthogonality and inner products of differential forms on $U$ are with respect to the inner products on $\mathscr{L}^{i}(U)$ and $\mathscr{L}^{p, q}(U)$.

Definition 1.23. Let

$$
\begin{aligned}
\mathscr{H}_{\bar{\partial}}^{0, q}= & \operatorname{kernel}\left(\bar{\partial}_{c}: \mathscr{A}_{c}^{0, q}(U) \rightarrow \mathscr{A}_{c}^{0, q+1}(U)\right) \\
& \cap\left\{w \in \mathscr{A}_{c}^{0, q}(U): w \perp \operatorname{range}\left(\bar{\partial}_{c}: \mathscr{A}_{c}^{0, q-1}(U) \rightarrow \mathscr{A}_{c}^{0, q}(U)\right)\right\} .
\end{aligned}
$$

Let

$$
\begin{aligned}
\mathscr{H}_{\bar{\partial}}^{2, q}= & \operatorname{kernel}\left(\bar{\partial}: \mathscr{A}^{2, q}(U) \rightarrow \mathscr{A}^{2, q+1}(U)\right) \\
& \cap\left\{w \in \mathscr{A}^{2, q}(U): w \perp \operatorname{range}\left(\bar{\partial}: \mathscr{A}^{2, q-1}(U) \rightarrow \mathscr{A}^{2, q}(U)\right)\right\} .
\end{aligned}
$$


Let

$$
\begin{aligned}
\mathscr{H}_{\bar{\partial}}^{1, q}= & \operatorname{kernel}\left(\bar{\partial}: \mathscr{D}^{1, q}(U) \rightarrow \mathscr{D}^{1, q+1}(U)\right) \\
& \cap\left\{w \in \mathscr{D}^{1, q}(U): w \perp \operatorname{range}\left(\bar{\partial}: \mathscr{D}^{1, q-1}(U) \rightarrow \mathscr{D}^{1, q}(U)\right)\right\} .
\end{aligned}
$$

Remark 1.24. The $\mathscr{H}_{\bar{\partial}}^{p}, q$ are defined only for $q \in\{0,1,2\}$. In defining them we use the conventions that spaces with a superscript $(p, r)$ where $r=-1$ or $r=3$ are $\{\overrightarrow{0}\}$ and that maps to or from such spaces are 0 -maps.

\section{Hodge DECOMPOSITION AND HARD LEFSCHETZ THEOREM}

In this section we state and prove the main theorem of this paper, Theorem 2.1. This theorem states that the $\bar{\partial}$-closed and $\bar{\partial}$-coclosed forms defined in Definition 1.23 provide a Hodge decomposition of $H_{(2)}^{*}(U)$ that agrees with the Hodge decomposition on the intersection homology of $X$ that appears in [CGM, §6.2]. Theorem 2.30 states that the hard Lefschetz theorem holds for the forms of Definition 1.23.

Theorem 2.1. The forms in $\mathscr{H}_{\bar{\partial}}^{p}, q$ are closed forms. The map resulting from considering their classes in $L^{2}$ cohomology induces an isomorphism

$$
\bigoplus_{p+q=i} \mathscr{H}_{\bar{\partial}}^{p, q} \cong H_{(2)}^{i}(U)
$$

for all $i$. The resulting $(p, q)$ decomposition of $H_{(2)}^{i}(U)$ is identified, via the isomorphism $H_{(2)}^{i}(U) \cong\left[I H_{i}(X)\right]^{*} \cong I H_{4-i}(X)$ with the Hodge $(p, q)$ decomposition of $I H_{*}(X)$ discussed in [CGM, $\left.\S 6.2\right]$.

Remark 2.2. A goal in this subject is to choose Dolbeault complexes whose $\bar{\partial}$ closed and $\bar{\partial}$-coclosed forms have the desirable relationship to $L^{2}$ cohomology described in the preceding theorem. Theorem 2.1 achieves this goal for normal surfaces. Ultimately one hopes to find such Dolbeault complexes in which the operators have closed ranges. It is likely that such complexes can be used to form a Hodge complex or a cohomological Hodge complex. Unfortunately in order to define a collection of $\bar{\partial}$-closed and $\bar{\partial}$-coclosed forms for which Theorem 2.1 is true, we have had to resort to a "second order" condition on the forms of type $(1, q)$. With this condition we have been unable to prove that our $\bar{\partial}$-operators have closed ranges. We believe that $L^{2}$ cohomology will eventually provide a beautiful and useful way to understand the Hodge decomposition on intersection homology. Theorem 2.1 provides strong evidence that this will be shown to be so once the relationships among domains of various differential operators on $U$ are better understood. It is important to note that the choice of domains for the operators in a Dolbeault complex has significance far beyond that of a mere formalism. For instance if one uses the maximal Dolbeault complex of forms of type $(0, q)$ or the minimal Dolbeault complex of forms of type $(2, q)$, one gets Dolbeault cohomology groups that do not have the desired relation to $L^{2}$ cohomology [Pa].

Previous work shows that a theorem analogous to Theorem 2.1 is true for singular curves, and it suggests some of the choices available in defining the Dolbeault complexes. The $L^{2}$ cohomology of the smooth part of a curve, in 
the metric induced from the ambient projective space, is isomorphic to the cohomology of the curve's desingularization. The harmonic forms representing the $L^{2}$ cohomology classes are exactly the $\bar{\partial}$-closed and $\bar{\partial}$-coclosed forms for the minimal $(0, q)$ and maximal $(1, q)$ Dolbeault complexes [H2]. Combining [H1] and [H2], one sees that the same complexes can be defined using the following domains for the Dolbeault operators: domain $\left(d^{0}\right)$, which equals domain $\left(d_{c}^{0}\right)$ because the induced metric on the curve is conical [CGM], and domain(restriction of $d^{1}$ to $(1,0)$-forms).

Proof of Theorem 2.1. The proof of Theorem 2.1 occupies most of the rest of this section. In outline the proof goes as follows. In [N2] we see that for $i \neq 2 \quad H_{(2)}^{i}(U) \cong \mathscr{H}^{i}=\bigoplus_{p+q=i} \mathscr{H}^{p, q}$. Using [H2], we show that $\mathscr{H}^{p, q}=$ $\mathscr{H}_{\bar{\partial}}^{p}, q$ for $(p, q)=(0,0),(0,1),(2,1)$, and $(2,2)$. We then show that $\mathscr{H}_{\bar{\partial}}^{0,2} \oplus \hat{\mathscr{H}}^{1,1} \oplus \mathscr{H}_{\bar{\partial}}^{2,0} \cong H_{(2)}^{2}(U)$, that $\mathscr{H}_{\bar{\partial}}^{1,0}=\mathscr{H}^{1,0}, \mathscr{H}_{\bar{\partial}}^{1,1}=* \hat{\mathscr{H}}^{1,1}$, and $\mathscr{H}_{\bar{\partial}}^{1,2}=\mathscr{H}^{1,2}$, and that $\mathscr{H}_{\bar{\partial}}^{2,0} \oplus \mathscr{H}_{\bar{\partial}}^{1,1} \oplus \mathscr{H}_{\bar{\partial}}^{0,2} \cong H_{(2)}^{2}(U)$. Finally, we show that the Hodge decomposition based on $\mathscr{H}_{\bar{\partial}}^{p}, q$ agrees with that of [CGM]. The last theorem of the section is the hard Lefschetz theorem.

The next two propositions collect results from [N2] and [H2]. Recall that $\tilde{X}$ has deRham cohomology groups $H^{i}(\tilde{X})$ and that there are many ways to define the groups $H^{p, q}(\tilde{X})$ [GrHa, p. 116].

Proposition 2.3 [N2, Theorem 1, Lemma 2.1, (2.7)]. (a) For $i \leq 1$

$$
H^{i}(\tilde{X}) \stackrel{\cong}{\longrightarrow} H_{(2)}^{i}(U) \cong \hat{\mathscr{H}}^{i}=\mathscr{H}^{i} \text {. }
$$

The first map is induced by restricting forms on $\tilde{X}$ to $\tilde{U}$ and identifying these forms with forms on $U$.

(b) For $i \geq 3$

$$
H^{i}(\tilde{X}) \cong H_{(2)}^{i}(U) \cong \hat{\mathscr{H}}^{i}=\mathscr{H}^{i} \text {. }
$$

(c) For $p+q=i$ with $i \leq 1$ or $i \geq 3$

$$
\mathscr{H}^{p, q}=\hat{\mathscr{H}}^{p, q} \cong H^{p, q}(\tilde{X}) .
$$

(d)

$$
\mathscr{H}^{1}=\mathscr{H}^{1,0} \oplus \mathscr{H}^{0,1}, \quad \mathscr{H}^{3}=\mathscr{H}^{2,1} \oplus \mathscr{H}^{1,2} .
$$

Proposition 2.4 [H2, Theorem 2.1 and analysis of adjoint operators]. (a) $\mathscr{H}_{\bar{\partial}}^{0, q} \cong$ $H^{0, q}(\tilde{X})$. The minimal $\bar{\partial}$-operators in the complex of $(0, q)$-forms have closed ranges.

(b) $\mathscr{H}_{\bar{\partial}}^{2, q} \cong H^{2, q}(\tilde{X})$.

Nagase proved that the cases $i=0$ and $i=4$ of Theorem 2.1 are true.

Proposition 2.5 [N2, Proposition 3.1].

$$
\hat{\mathscr{H}}^{0}=\mathscr{H}_{\bar{\partial}}^{0,0}, \quad \hat{\mathscr{H}}^{4}=\mathscr{H}_{\bar{\partial}}^{2,2} .
$$

In fact we know more about the actions of exterior differentation and Dolbeault operators on functions. 
Lemma 2.6. domain $\left(\bar{\partial}_{c}^{0,0}\right)=\operatorname{domain}\left(d_{c}^{0}\right)$.

Proof. Let $\left\langle\ldots, \ldots\right.$ denote the $L^{2}$ inner products on functions and forms on $U$. A sequence of compactly supported functions $\left\{f_{i}\right\}$ represents an element of domain $\left(\bar{\partial}_{c}^{0,0}\right)$, respectively domain $\left(d_{c}^{0}\right)$, if and only if the sequences $\left\{f_{i}\right\}$ and $\left\{\bar{\partial} f_{i}\right\}$, respectively $\left\{f_{i}\right\}$ and $\left\{d f_{i}\right\}$, are Cauchy. Because every $F_{i j}=f_{i}-f_{j}$ is compactly supported and because $U$ is Kähler, we have the following equations, which ensure that the sequences for $\bar{\partial}$ are Cauchy if and only if the sequences for $d$ are Cauchy.

$$
\left\langle\bar{\partial} F_{i j}, \bar{\partial} F_{i j}\right\rangle=\left\langle\bar{\partial}^{*} \bar{\partial} F_{i j}, F_{i j}\right\rangle=\frac{1}{2}\left\langle d^{*} d F_{i j}, F_{i j}\right\rangle=\frac{1}{2}\left\langle d F_{i j}, d F_{i j}\right\rangle .
$$

Lemma 2.7 [N1, Assertion A]. $d_{c}^{0}=d^{0}$.

Also, a good deal is known about the kernels of closed extensions of minimal exterior differentiation and their adjoints. Recall that our indexing of adjoints differs from Nagase's.

Lemma $2.8\left[\mathrm{~N} 2\right.$, Lemma 2.1]. If $i \neq 2, \operatorname{kernel}\left(d_{c}^{i}: A_{c}^{i}(U) \rightarrow \mathscr{L}^{i+1}(U)\right)=$ $\operatorname{kernel}\left(d^{i}: A^{i}(U) \rightarrow \mathscr{L}^{i+1}(U)\right)$, and $\left\{w \in \mathscr{L}^{i}(U): w\right.$ smooth and $\delta_{c}^{i}(w)=$ $0\}=\left\{w \in \mathscr{L}^{i}(U): w\right.$ smooth and $\left.\delta^{i}(w)=0\right\}$.

Proposition 2.9. $\hat{\mathscr{H}}^{0,1}=\mathscr{H}_{\bar{\partial}}^{0,1}$.

Proof. Lemmas 2.6 and 2.7 show that $\left\{(0,1)\right.$-forms orthogonal to range $\left.\left(\bar{\partial}_{c}\right)\right\}=$ $\{(0,1)$-forms orthogonal to range $(d)\}$. Lemma 2.8 shows that any smooth $(0,1)$-form in kernel $(d)$ is in kernel $\left(d_{c}\right)$ and thus is in kernel $\left(\bar{\partial}_{c}\right)$. It follows that $\hat{\mathscr{H}}^{0,1} \subset \mathscr{H}_{\bar{\partial}}^{0,1}$. By Proposition 2.3(c) and Proposition 2.4, $\hat{\mathscr{H}}^{0,1}$ and $\mathscr{H}_{\bar{\partial}}^{0,1}$ have the same dimension.

Proposition 2.10. $\hat{\mathscr{H}}^{2,1}=\mathscr{H}_{\bar{\partial}}^{2,1}$.

Proof. Take adjoints in the proof of the preceding proposition. (This involves applying the Hodge *-operator and changing domains from $\mathscr{A}_{c}^{p, q}(U)$ to $\left.\mathscr{A}^{n-p, n-q}(U).\right) \quad \operatorname{Kernel}\left(\bar{\partial}^{2,1}\right)=\{(2,1)$-forms in $\operatorname{kernel}(d)\}$, and $\{(2,1)$ forms orthogonal to range $(d)\} \subset\{(2,1)$-forms orthogonal to range $(\bar{\partial})\}$. It follows that $\hat{\mathscr{H}}^{2,1} \subset \mathscr{H}_{\bar{\partial}}^{2,1}$; and Propositions $2.3(\mathrm{c})$ and 2.4 show that $\hat{\mathscr{H}}^{2,1}$ and $\mathscr{H}_{\bar{\partial}}^{2,1}$ have the same dimension.

Because $\mathscr{H}_{\bar{\partial}}^{2,0}$ and $\mathscr{H}_{\bar{\partial}}^{0,2}$ are exactly the forms on $U$ that are identified with the restrictions to $\tilde{U}$ of harmonic $(2,0)$, respectively $(0,2)$, forms on $\tilde{X}$ [H2, Proposition 2.3 and its proof], there is a map $\mathscr{H}_{\bar{\partial}}^{2,0} \oplus \mathscr{H}_{\bar{\partial}}^{0,2} \longrightarrow H_{(2)}^{2}(U)$.

Proposition 2.11. The above map is injective.

Proof. Recall from the beginning of this section the labeling of sets that appear in the desingularization $\pi: \tilde{X} \rightarrow X$. Let $E=\bigcup_{i=1}^{k} E_{i}$ realize $E$ as a union of smooth irreducible curves. Let $\tilde{N}_{i}$ be a sufficiently small tubular neighborhood of $E_{i}$. Let $\tilde{N}=\bigcup_{i=1}^{k} \tilde{N}_{i}$. The closure of $\tilde{N}$ is topologically a manifold with boundary. 
The proof of this proposition relies on the cohomological Mayer-Vietoris sequence associated to $\tilde{X}=\tilde{N} \cup \tilde{U}$ and on the cohomological sequence associated to the pair $(\tilde{N}, \tilde{N} \cap \tilde{U})$.

$$
\begin{aligned}
& \cdots \rightarrow H^{1}(\tilde{N} \cap \tilde{U}) \stackrel{h}{\rightarrow} H^{2}(\tilde{X}) \stackrel{r_{1} \oplus r_{2}}{\rightarrow} H^{2}(\tilde{N}) \oplus H^{2}(\tilde{U}) \stackrel{r_{3}}{\rightarrow} H^{2}(\tilde{N} \cap \tilde{U}) \rightarrow \cdots \\
& g \searrow \quad \uparrow_{f} \quad \| \oplus 0 \\
& \cdots \rightarrow H^{2}(\tilde{N}, \tilde{N} \cap \tilde{U}) \quad \rightarrow \quad H^{2}(\tilde{N}) \quad \rightarrow H^{2}(\tilde{N} \cap \tilde{U}) \rightarrow \cdots
\end{aligned}
$$

Identifying $H^{2}(\tilde{N}, \tilde{N} \cap \tilde{U})$ with cohomology with compact supports $H_{c}^{2}(\tilde{N})$, we define $f: H^{2}(\tilde{N}, \tilde{N} \cap \tilde{U})=H_{c}^{2}(\tilde{N}) \rightarrow H^{2}(\tilde{X})$ via the identity map on these compactly supported differential forms. Using the construction of $h$ at the level of differential forms, we see that there is a factorization $h=f \circ g$. Before completing the proof of the proposition, we need a lemma and its proof.

Lemma 2.13. $H^{2}(\tilde{N}, \tilde{N} \cap \tilde{U})$ is generated by the Thom classes associated to the bundles $\tilde{N}_{i} \rightarrow E_{i}$. The images under $f$ of these Thom classes have harmonic representatives of type $(1,1)$.

Proof. $H^{2}(\tilde{N}, \tilde{N} \cap \tilde{U}) \stackrel{\cong}{\longrightarrow} H_{2}(\tilde{N}) \stackrel{\cong}{\longrightarrow} \bigoplus_{i} H_{2}\left(\tilde{N}_{i}\right) \cong \bigoplus_{i} H^{2}\left(\tilde{N}_{i}, \tilde{N}_{i} \cap\left(\tilde{X} \backslash E_{i}\right)\right)$.

The maps arise from Lefschetz duality, Mayer-Vietoris for $\tilde{N}=\bigcup_{i} \tilde{N}_{i}$, and Lefschetz duality. The characterization of the groups appearing in the final direct sum is part of the Thom isomorphism theorem. See, e.g., [GrHa, p. 163] for the description of the images under $f$ of these Thom classes.

We now conclude the proof of Proposition 2.11 by showing that any element of $\mathscr{H}^{2,0}(\tilde{X}) \oplus \mathscr{H}^{0,2}(\tilde{X})$ maps by restriction to a nonzero element of $H^{2}(\tilde{U})$. Suppose $\alpha \in \operatorname{kernel}\left(r_{2}\right)$ in diagram (2.12). Then $r_{1}(\alpha) \in \operatorname{kernel}\left(r_{3}\right)$. It follows from the commutativity of (2.12) that $\alpha \in \operatorname{image}(f)$. By Lemma $2.13 \alpha$ has a representative of type $(1,1)$.

Because harmonic forms are orthogonal to the image of exterior differentiation on $\tilde{X}$, no nonzero element of $\mathscr{H}^{2,0}(\tilde{X}) \oplus \mathscr{H}^{0,2}(\tilde{X})$ has a representative of type $(1,1)$. Thus no nonzero element of $\mathscr{H}^{2,0}(\tilde{X}) \oplus \mathscr{H}^{0,2}(\tilde{X})$ is in $\operatorname{kernel}\left(r_{2}\right)$, and so the restriction of every nonzero element of $\mathscr{H}^{2,0}(\tilde{X}) \oplus \mathscr{H}^{0,2}(\tilde{X})$ to $\tilde{U}$ lies outside the image of exterior differentiation on $\tilde{U}$. Thus the nonzero elements of $\mathscr{H}_{\tilde{\delta}}^{2,0} \oplus \mathscr{H}_{\tilde{\delta}}^{0,2}$ define nonzero elements of $H_{(2)}^{2}(U)$, which completes the proof of Proposition 2.11.

We now proceed to describe the rest of $H_{(2)}^{2}(U)$.

Proposition 2.14. The map $\hat{\mathscr{H}}^{1,1} \oplus \mathscr{H}_{\tilde{\partial}}^{2,0} \oplus \mathscr{H}_{\partial}^{0,2} \rightarrow H_{(2)}^{2}(U)$ defined by taking $d$-closed forms to their $L^{2}$ cohomology classes is an isomorphism.

Proof. To prove injectivity of the map, suppose $w^{1,1}+h=d \beta$, where $w^{1,1} \in$ $\hat{\mathscr{H}}^{1,1}, h \in \mathscr{H}_{\tilde{\partial}}^{2,0} \oplus \mathscr{H}_{\bar{\partial}}^{0,2}$, and $\beta \in \operatorname{domain}\left(d^{1}\right)$. Recall that $\langle-,-\rangle$ is the $L^{2}$ inner product on forms on $U$.

$$
\left\langle w^{1,1}, w^{1,1}\right\rangle=\left\langle w^{1,1}, w^{1,1}+h\right\rangle=\left\langle w^{1,1}, d \beta\right\rangle=0 .
$$

It follows that $w^{1,1}=0$. Then Proposition 2.11 implies that $h=0$ also.

To prove surjectivity of the map in the proposition, we use two lemmas. 
Lemma 2.15. (a) $\mathscr{H}^{0,2}=\mathscr{H}_{\bar{\partial}}^{0,2}$.

(b) $\mathscr{H}^{2,0}=\mathscr{H}_{\bar{\partial}}^{2,0}$.

Proof. (a) By [H2, Proposition 2.3 and its proof] the isomorphisms $\mathscr{L}^{0,2}(\tilde{X}) \cong$ $\mathscr{L}^{0,2}(\tilde{U}) \cong \mathscr{L}^{0,2}(U)$ identify $\mathscr{H}^{0,2}(\tilde{X})$ with $\mathscr{H}_{\bar{\partial}}^{0,2}$. Therefore, $x \in \mathscr{H}_{\bar{\partial}}^{0,2} \Rightarrow$ $x$ is a $(0,2)$-form and $x \in \operatorname{kernel}(d) \cap \operatorname{kernel}(* d *) \Rightarrow x \in \mathscr{H}^{0,2}$. On the other hand, $x \in \mathscr{H}^{0,2} \Rightarrow x$ is a $(0,2)$-form and $x$ is orthogonal to the image under $d$ of the smooth compactly supported 1 -forms on $U \Rightarrow x$ is a $(0,2)$-form and $x$ is orthogonal to the image under $\bar{\partial}$ of the smooth compactly supported $(0,1)$-forms on $U \Rightarrow x \in \mathscr{H}_{\bar{\partial}}^{0,2}$.

Because [N2, Theorem 1], respectively [H2, proof of Proposition 2.3], shows that $\mathscr{H}^{2,0}=\overline{\mathscr{H}}^{0,2}$, resp. $\mathscr{H}_{\bar{\partial}}^{2,0}=\overline{\mathscr{H}}_{\bar{\partial}}^{0,2}$, we see that $(\mathrm{a}) \Rightarrow$ (b).

Lemma 2.16. There is an antilinear bijection from $\hat{\mathscr{H}}^{2}$ to the dual of $H_{(2)}^{2}(U)$. Proof. The map $\hat{\mathscr{H}}^{2} \rightarrow H_{(2)}^{2}(U)^{*}$ arises from the pairing $H_{(2)}^{2}(U) \times \hat{\mathscr{H}}^{2} \rightarrow \mathbb{C}$ given by $([y], h) \rightarrow\langle y, h\rangle$. This pairing is well-defined because $\langle d x, h\rangle=$ $\left\langle x, d^{*} h\right\rangle=\langle x, \overrightarrow{0}\rangle=0$. The map is injective because for any $h \in \hat{\mathscr{H}}^{2}$ we can consider $\langle[h], h\rangle=\langle h, h\rangle=\|h\|^{2}$. An antilinear injection between vector spaces of the same finite dimension is a bijection.

To prove surjectivity of the map in the proposition, let $h$ be an arbitrary element of $\hat{\mathscr{H}}^{2}$ satisfying

$$
\forall y \in \mathscr{H}_{\bar{\partial}}^{2,0} \oplus \mathscr{H}_{\bar{\partial}}^{0,2}, \quad\langle[y], h\rangle=0 .
$$

Write $h=h^{2,0}+h^{1,1}+h^{0,2}$, where $h^{p, q} \in \mathscr{H}^{p, q}$. By Lemma 2.15, (2.17) implies that $h^{2,0}$ and $h^{0,2}$ are zero, i.e. that $h^{1,1}=h \in \mathscr{H}^{1,1} \cap \hat{\mathscr{H}}^{2}=\hat{\mathscr{H}}^{1,1}$. A dimension count then implies that the injective map $\hat{\mathscr{H}}^{1,1} \oplus \mathscr{H}_{\bar{\partial}}^{2,0} \oplus \mathscr{H}_{\bar{\partial}}^{0,2} \rightarrow$ $H_{(2)}^{2}(U)$ is surjective, which completes the proof of Proposition 2.14.

We now describe $\mathscr{H}_{\bar{\partial}}^{1, q}$ for $q \in\{0,1,2\}$. In the proof we use the notational convention of Remark 1.24.

Proposition 2.18. (a) $\mathscr{H}_{\bar{\partial}}^{1,0}=\hat{\mathscr{H}}^{1,0}$.

(b) $\mathscr{H}_{\bar{\partial}}^{1,1}=* \hat{\mathscr{H}}^{1,1}$.

(c) $\mathscr{H}_{\bar{\partial}}^{1,2}=\hat{\mathscr{H}}^{1,2}$.

Proof. We actually show $\mathscr{H}_{\bar{\partial}}^{1, q}=\mathscr{L}^{1, q}(U) \cap \operatorname{kernel}\left(d_{c}\right) \cap \operatorname{kernel}(* d *)$. (Note that $\operatorname{kernel}(* d *)=\operatorname{kernel}\left(d_{c}^{*}\right)$.) An application of Lemma 2.8 shows that for $q \neq 1$ this result is equivalent to what is stated in the proposition. It follows directly from definitions that for $q=1$ this result is equivalent to what is stated in the proposition.

Observe that $w \in \mathscr{L}^{1, q}(U) \cap \operatorname{kernel}\left(d_{c}\right) \cap \operatorname{kernel}\left(d_{c}^{*}\right)$ implies $w \in$ $\mathscr{L}^{1, q}(U) \cap$ domain $\left(d_{c} d_{c}^{*}+d_{c}^{*} d_{c}\right), d w=0$, and $w \perp$ image under $d$ of the smooth forms compactly supported on $U$. It follows that $w \in \mathscr{L}^{1, q}(U), \bar{\partial} w=$ 0 , and $w \perp$ image under $\bar{\partial}$ of the smooth forms compactly supported on $U$. 
Because domain $\left(d_{c} d_{c}^{*}+d_{c}^{*} d_{c}\right) \subset$ domain $\left(d_{c}\right)$, it follows that $w \perp$ image under $\bar{\partial}$ of domain $\left(d_{c} d_{c}^{*}+d_{c}^{*} d_{c}\right) \cap \mathscr{L}^{1, q-1}(U)$. Thus $w \in \mathscr{H}_{\bar{\partial}}^{1, q}$.

On the other hand suppose $w \in \mathscr{H}_{\bar{\partial}}^{1, q}$. Then

$$
w \in \mathscr{L}^{1, q}(U) \cap \operatorname{domain}\left(d_{c} d_{c}^{*}+d_{c}^{*} d_{c}\right), \bar{\partial} w=0,
$$

and $w \perp$ image under $\bar{\partial}$ of smooth forms compactly supported on $U$. It follows that $* \bar{\partial} * w=0$. Thus $w$ is a smooth form in domain $\left(d_{c} d_{c}^{*}+d_{c}^{*} d_{c}\right)$ that satisfies $(\bar{\partial} * \bar{\partial} *+* \bar{\partial} * \bar{\partial}) w=0$ when the differentiation is done formally. Because $U$ is Kähler, $w \in \operatorname{kernel}\left(d_{c} d_{c}^{*}+d_{c}^{*} d_{c}\right)$, from which it follows that $w \in \mathscr{L}^{1, q}(U) \cap \operatorname{kernel}\left(d_{c}\right) \cap \operatorname{kernel}\left(d_{c}^{*}\right)$.

We now complete the description of our $(p, q)$ decomposition of $H_{(2)}^{2}(U)$. Because $\mathscr{H}_{\bar{\partial}}^{1,1}=* \hat{\mathscr{H}}^{1,1} \subset \mathscr{H}^{1,1}$, the elements of $\mathscr{H}_{\bar{\partial}}^{1,1}$ are $d$-closed.

Proposition 2.19. The map $\mathscr{H}_{\bar{\partial}}^{1,1} \oplus \mathscr{H}_{\bar{\partial}}^{2,0} \oplus \mathscr{H}_{\bar{\partial}}^{0,2} \rightarrow H_{(2)}^{2}(U)$ defined by taking $d$-closed forms to their $L^{2}$ cohomology classes is an isomorphism.

Proof. Our study of $H_{(2)}^{2}(U)$ uses the analysis of $I_{2}(X)$ appearing in [CGM]. Recall that integration identifies $H_{(2)}^{2}(U)$ with the dual of $I H_{2}(X)$. [HsP, N3]

$$
I H_{2}(X) \cong \text { Image }\left(H_{2}(U) \rightarrow H_{2}(X)\right)
$$

where the map $H_{2}(U) \rightarrow H_{2}(X)$ is induced from the inclusion of $U$ in $X$. Use $i$ to denote the inclusion of $\tilde{U}$ in $\tilde{X}$. There is a commutative diagram

$$
\begin{array}{ll}
H_{2}(\tilde{U}) \stackrel{i_{*}}{\longrightarrow} & H_{2}(\tilde{X}) \\
\pi_{*} \mid \| l & \downarrow \pi_{*} \\
H_{2}(U) \longrightarrow H_{2}(X) .
\end{array}
$$

Denote by $H_{2,0}(\tilde{X}) \oplus H_{0,2}(\tilde{X})$ the subspace of $H_{2}(\tilde{X})$ consisting of classes whose integration pairing with every element of $\mathscr{H}^{1,1}(\tilde{X})$ is zero. $H_{2,0}(\tilde{X}) \oplus$ $H_{0,2}(\tilde{X})$ lies in the image of $i_{*}$ [CGM].

By Proposition 2.14 and Proposition 2.18(b) $\mathscr{H}_{\bar{\partial}}^{1,1}$ and $\hat{\mathscr{H}}^{1,1}$ are vector spaces of the same finite dimension. The following three lemmas, combined with Proposition 2.14, prove Proposition 2.19.

Lemma 2.20 [H2, Proposition 2.3 and its proof]. (a) $\pi^{*}\left(\mathscr{H}_{\bar{\partial}}^{2,0}\right)=\left.\mathscr{H}^{2,0}(\tilde{X})\right|_{\tilde{U}}$.

(b) $\pi^{*}\left(\mathscr{H}_{\bar{\partial}}^{0,2}\right)=\left.\mathscr{H}^{0,2}(\tilde{X})\right|_{\tilde{U}}$.

Lemma 2.21. For any $h \in \mathscr{H}_{\bar{\partial}}^{1,1}$ and any $[z] \in \pi_{*}\left(H_{2,0}(\tilde{X}) \oplus H_{0,2}(\tilde{X})\right)$, the integration pairing of $h$ with $[z]$ gives zero.

Proof. Assume $h \in \mathscr{H}_{\bar{\partial}}^{1,1}$. Because [z] is the image of a homology class on $U$, we may assume the cycle $z$ is a cycle on $U$. Poincaré duality (see, e.g., [BoT]) shows that there is a smooth form $\alpha$ compactly supported in $U$ such that $\int_{z} h=\int_{U} \alpha \wedge h$.

Denote by $i_{*}$ the map that takes smooth forms compactly supported on $\tilde{U}$ to smooth forms on $\tilde{X}$ by extension by zero. There is an $\tilde{\eta} \in \mathscr{H}^{2,0}(\tilde{X}) \oplus \mathscr{H}^{0,2}(\tilde{X})$ 
and a $\tilde{\beta} \in \mathscr{L}^{1}(\tilde{X})$ such that $d \tilde{\beta}=i_{*}\left(\pi^{*} \alpha\right)-\tilde{\eta}$. Calculations with the metrics (see, e.g., [H2]) show that $\left.\mathscr{L}^{1}(\tilde{X})\right|_{\tilde{U}} \subset \pi^{*}\left(\mathscr{L}^{1}(U)\right)$. Let $\beta$ denote the element of $\mathscr{L}^{1}(U)$ satisfying $\left.\tilde{\beta}\right|_{\tilde{U}}=\pi^{*} \beta$. By Lemma 2.20 there is a form $\eta$ satisfying $\left.\tilde{\eta}\right|_{\tilde{U}}=\pi^{*} \eta$. Then $d \beta=\alpha-\eta$.

It follows that $\int_{z} h=\int_{U} \alpha \wedge h=\int_{U} \eta \wedge h+\int_{U} d \beta \wedge h$. Checking $(p, q)$ types shows that $\int_{U} \eta \wedge h=0$. Because $h=* \xi$ for some $\xi \in \hat{\mathscr{H}}^{1,1}$ and because $\xi \perp$ range $(d), \int_{U} d \beta \wedge h=\int_{U} d \beta \wedge * \xi=<d \beta, \xi>=0$.

Lemma 2.22. For any nonzero $h \in \mathscr{H}_{\bar{\partial}}^{1,1}$ there is a class in $I_{2}(X)$ for which the integration pairing with $h$ is nonzero. In particular, when this class is represented by a cycle on $U$, Stokes' theorem applied on the cycle shows that $h \notin \operatorname{range}(d)$. Proof. Any nonzero $h \in \mathscr{H}_{\bar{\partial}}^{1,1}$ is $* \xi$ for some nonzero $\xi \in \hat{\mathscr{H}}^{1,1}$. Because such a $\xi$ represents a nonzero class in $H_{(2)}^{2}(U)$, there is a class $[y] \in I H_{2}(X)$ satisfying: for any $[z] \in I H_{2}(X)$ the intersection pairing of $[z]$ with $[y]$ equals $\int_{z} \xi[\mathrm{CGM}]$. By Poincare duality (see, e.g., [BoT]) there is a $d$-closed smooth form $\alpha$ compactly supported on $U$ satisfying the properties: (a) for all $[z] \epsilon$ $I H_{2}(X) \int_{z} \xi=\int_{z} \alpha$; and (b) for closed forms $\mu \int_{y} \mu=\int_{U} \alpha \wedge \mu$. (b)

By property (a) there is a $\beta \in \mathscr{L}^{1}(U)$ such that $d \beta=\alpha-\xi$. By property

$$
\begin{aligned}
\int_{y} h & =\int_{y} * \xi=\int_{y} \alpha \wedge * \xi=\int_{y}(\xi+d \beta) \wedge * \xi \\
& =\int_{y} \xi \wedge * \xi+\int_{y} d \beta \wedge * \xi=\|\xi\|^{2}+\langle d \beta, \xi\rangle=\|\xi\|^{2} \neq 0 .
\end{aligned}
$$

We now compare the decomposition $H_{(2)}^{i}(U)=\bigoplus_{p+q=i} \mathscr{H}_{\bar{\partial}}^{p, q}$ with the Hodge decomposition of intersection homology described in [CGM]. Recall that [CGM] assigns a Hodge structure to $I H_{*}(X)$ as follows. First a class in $H_{*}(\tilde{X})$ is of type $(p, q)$ if its Poincaré dual is of type $(2-p, 2-q)$, i.e., if its hom dual is of type $(p, q)$. Second $I H_{*}(X)$ is identified with a subset of $H_{*}(\tilde{X})$, and it is shown that the Hodge structure on $H_{*}(\tilde{X})$ restricts to give a Hodge structure on this subset. Then a class in $I H_{*}(X)$ is assigned the type of the corresponding class in $H_{*}(\tilde{X})$.

The Hodge structure on $I H_{*}(X)$ determines a Hodge structure on $H_{(2)}^{*}(U)$ via the diagram

$$
I H_{k}(X) \stackrel{\cong}{I} \operatorname{Hom}\left(I H_{4-k}(X), \mathbb{C}\right) \underset{J}{\stackrel{\cong}{\leftrightarrows}} H_{(2)}^{4-k}(U) .
$$

$I$ arises from the intersection pairing, and $J$ arises from integration. Assign $[h] \in H_{(2)}^{4-k}(U)$ type $(p, q)$ when $I^{-1}(J([h]))$ has type $(2-p, 2-q)$.

Proposition 2.24. The $(p, q)$ decomposition of $L^{2}$ cohomology described in this paper agrees with that described for intersection homology in [CGM], i.e., an $L^{2}$ cohomology class is represented by $h \in \mathscr{H}_{\bar{\partial}}^{p}, q$ if and only if its image under $I^{-1} \circ J$ is in $I_{2-p, 2-q}(X)$.

Proof. There can be no disagreement about the pure Hodge decompositions of $H_{(2)}^{0}(U)$ and $H_{(2)}^{4}(U)$. 
$H_{(2)}^{1}(U)$ : To begin the identification of the two decompositions of $H_{(2)}^{1}(U)$, we need three lemmas.

Lemma 2.25. range $(d) \cap \mathscr{L}^{0,1}(U)=\{\overrightarrow{0}\}$.

Proof. By Lemma 2.7 it suffices to show that range $\left(d_{c}\right) \cap \mathscr{L}^{0,1}(U)=\{\overrightarrow{0}\}$. Suppose $d_{c} f=x \in \mathscr{L}^{0,1}(U)$. Then $\partial_{c} f=0$, i.e., $\bar{\partial}_{c} \bar{f}=0$. By [H2, Proposition 2.2 and its proof] $f$ is constant and so $x=\overrightarrow{0}$.

Lemma 2.26. $\hat{\mathscr{H}}^{0,1}=\operatorname{kernel}(d) \cap \mathscr{L}^{0,1}(U)$.

Proof. Apply the preceding lemma and Theorem 1 of [N2].

Lemma 2.27. $\pi^{*}\left(\mathscr{H}_{\tilde{\partial}}^{0,1}\right)=\left.\mathscr{H}^{0,1}(\tilde{X})\right|_{\dot{U}}$.

Proof. By comparison of metrics (see, e.g., [H2]) $\left.\mathscr{H}^{0,1}(\tilde{X})\right|_{\tilde{U}} \subset \pi^{*}\left(\mathscr{L}^{0,1}(U)\right)$. Thus $\left.\mathscr{H}^{0,1}(\tilde{X})\right|_{\tilde{U}} \subset \pi^{*}(\operatorname{kernel}(d))$. This observation, followed by Lemma 2.26 and Proposition 2.9, gives

$$
\left.\mathscr{H}^{0,1}(\tilde{X})\right|_{\tilde{U}} \hookrightarrow \pi^{*}\left(\operatorname{ker}(d) \cap \mathscr{L}^{0,1}(U)\right)=\pi^{*}\left(\hat{\mathscr{H}}^{0,1}\right)=\pi^{*}\left(\mathscr{H}_{\bar{\partial}}^{0,1}\right) .
$$

By [H2, Theorem 2.1] the vector spaces at the ends have the same finite dimension. Thus they are equal.

To complete the analysis of the decomposition of $H_{(2)}^{1}(U)$, let $i: \tilde{U} \hookrightarrow \tilde{X}$ and $i_{*}: H_{*}(\tilde{U}) \rightarrow H_{*}(\tilde{X})$. The decomposition in [CGM] is based on the diagram.

$$
H_{1}(\tilde{X}) \underset{i_{*}}{\cong} H_{1}(\tilde{U}) \underset{\pi_{*}}{\stackrel{\cong}{\rightrightarrows}} H_{1}(U) \cong I H_{1}(X) .
$$

Let $\left\{\sigma_{k}\right\}$ be a set of cycles on $\tilde{U}$ whose images under $i_{*}$ span the subset of $H_{1}(\tilde{X})$ composed of classes of type $(1,0)$. Thus for every $h \in \mathscr{H}^{0,1}(\tilde{X}), \int_{\sigma_{k}} h$ $=0$; and any 1-cycle $\sigma$ satisfying $\int_{\sigma} h=0$ for all $h \in \mathscr{H}^{0,1}(\tilde{X})$ is homologous to a linear combination of elements of $\left\{\sigma_{k}\right\}$. The decomposition of $H_{(2)}^{1}(U)$ induced by the [CGM] decomposition of $I H_{1}(X)$ assigns $\xi \in \hat{\mathscr{P}}^{1}$ type $(0,1)$ if $\int_{\sigma_{k}} \pi^{*} \xi=0$ for all $k$. For all $\xi \in \mathscr{H}_{\bar{\partial}}^{0,1} \int_{\sigma_{k}} \pi^{*} \xi$, equals zero by Lemma 2.27. Thus [CGM] assigns type $(0,1)$ to elements of $\mathscr{H}_{\bar{\partial}}^{0,1}$. Switching the roles of 0 and 1 , we see that similar arguments show that [CGM] assigns type $(1,0)$ to elements of $\mathscr{H}_{\bar{\partial}}^{1,0}$.

$H_{(2)}^{3}(U)$ : The reasoning relating the decompositions of $H_{(2)}^{3}(U)$ is parallel to that used for $H_{(2)}^{1}(U)$. This time [CGM] uses

$$
H_{3}(\tilde{X}) \underset{\pi_{*}}{\stackrel{\cong}{\rightrightarrows}} H_{3}(X) \cong I H_{3}(X) .
$$

The three lemmas are replaced by the following lemma, based largely on [H2].

Lemma 2.28. Each element of $\pi^{*}\left(\mathscr{H}_{\bar{\partial}}^{2,1}\right)$ can be viewed as a closed $L^{2}$ form on $\tilde{X}$. As $L^{2}$ forms on $\tilde{X}$ these are cohomologous to the elements of $\mathscr{H}^{2,1}(\tilde{X})$. The elements of $\pi^{*}\left(\mathscr{H}_{\bar{\partial}}^{2,1}\right)$ are smooth on $\tilde{U}$ and bounded in the pointwise norm associated with the restriction of $\tilde{X}$ 's metric to $\tilde{U}$.

Proof. The first two sentences of the lemma follow from reasoning based on the commutative diagram in the proof of Proposition 2.6 of [H2]. The construction of the diagram and its relationship with cohomology on $\tilde{X}$ are based 
on Propositions 1.16 and 1.17 of [H2]. Finally Lemma 2.27 of the current paper identifies $\pi^{*}\left(\mathscr{H}_{\bar{\partial}}^{0,1}\right)$ with a set of forms that extend smoothly to $\tilde{X}$. Thus these forms are bounded in the pointwise norm associated with $\tilde{X}$ 's metric. $\mathscr{H}_{\bar{\partial}}^{2,1}=* \mathscr{H}_{\bar{\partial}}^{0,1}$, where $*$ denotes the Hodge $*$-operator based on the metric on $U$. Explicit calculations with this $*$-operator (as a map $\mathscr{H}_{\bar{\partial}}^{0,1} \rightarrow \mathscr{H}_{\bar{\partial}}^{2,1}$ it will be pointwise norm-decreasing for the norm based on $\tilde{X}$ 's metric) finish the proof of the third sentence of the lemma.

To carry out the integration pairing, one can realize homology using p.l. geometric chains.

$H_{(2)}^{2}(U)$ : The comparison of decompositions of $H_{(2)}^{2}(U)$ follows from the observations introducing the proof of Proposition 2.19 and from Lemmas 2.20 and 2.21 .

This completes the proof of Proposition 2.24 and the proof of Theorem 2.1.

We now state and prove the $L^{2}$ version of the hard Lefschetz theorem in this setting. Let $w$ be the Kähler form on $U$, and let $\mathscr{H}_{\bar{\partial}}^{i}=\bigoplus_{p+q=i} \mathscr{H}_{\bar{\partial}}^{p}, q$. For $\alpha \in \mathscr{H}_{\bar{\partial}}^{i}$, let $L(\alpha)=\alpha \wedge w$.

Definition 2.29. (a) $P^{2-k}=\operatorname{kernel}\left(L^{k+1}: \mathscr{H}_{\bar{\partial}}^{2-k} \rightarrow \mathscr{H}_{\bar{\partial}}^{4+k}\right)$.

(b) $P^{p, q}=P^{p+q} \cap \mathscr{H}_{\bar{\partial}}^{p, q}$.

Theorem 2.30. (a) $L^{k}: \mathscr{H}_{\bar{\partial}}^{2-k} \cong \mathscr{H}_{\bar{\partial}}^{2+k}$.

(b) $\mathscr{H}_{\bar{\partial}}^{i}=\bigoplus_{k} L^{k} P^{i-2 k}$.

(c) $P^{i}=\bigoplus_{p+q=i} P^{p, q}$.

Proof. For all $i \mathscr{H}_{\bar{\partial}}^{i} \subset \mathscr{H}^{i}$, and for $i \neq 2 \mathscr{H}_{\bar{\partial}}^{i}=\mathscr{H}^{i}$. For $p+q \neq 2$ $\mathscr{H}_{\bar{\partial}}^{p, q}=\mathscr{H}^{p, q} \cdot \mathscr{H}_{\bar{\partial}}^{2,0}=\mathscr{H}^{2,0}, \mathscr{H}_{\bar{\partial}}^{0,2}=\mathscr{H}^{0,2}$, and $\mathscr{H}_{\bar{\partial}}^{1,1}=* \hat{\mathscr{H}}^{1,1}$. Thus the proof of [N2, Theorem 2], including the assertion that $L$ maps harmonic forms to harmonic forms, becomes a proof of this theorem once we have shown that $w$ lies not only in $\mathscr{H}^{1,1}$ but actually in $* \hat{\mathscr{H}}^{1,1}$. Because $w=* w$ [N2, (7.2)], it suffices to show that $w \in \hat{\mathscr{H}}^{1,1}$.

Because $w \in \mathscr{L}^{2}(U)$ and because $d w=0$, it suffices to show that $w \perp$ range $(d)$. In determining range $\left(d^{1}: \mathscr{L}^{1}(U) \rightarrow \mathscr{L}^{2}(U)\right)$, it suffices to consider $\psi \in \mathscr{L}^{1}(U)$ for which $\psi \perp$ range $\left(d^{0}\right)$, i.e., $\psi \in \mathscr{L}^{1}(U)$ satisfying $\psi \in$ $\operatorname{kernel}\left(* d_{c} *\right)$. Because $w=* w$ and because $\psi \wedge w=-\sqrt{-1} * \bar{\psi}$ [N2], we see that for $\psi \in \operatorname{domain}(d) \cap \operatorname{kernel}\left(* d_{c} *\right)$,

$$
0=d(\psi \wedge w)=d \psi \wedge w-\psi \wedge d w=d \psi \wedge w
$$

Thus, $0=\int_{U} d \psi \wedge w$, which shows that $w=* w$ is orthogonal to range $(d)$.

\section{REFERENCES}

[B] A. Borel, $L^{2}$-cohomology and intersection cohomology of certain arithmetic varieties, Emmy Noether at Bryn Mawr (J. Sally and B. Srinivasan, eds.), Springer-Verlag, Berlin, Heidelberg, and New York, 1983, pp. 119-131. 
[BCa] A. Borel and W. Casselman, Cohomologie d'intersection et $L^{2}$-cohomologie de variétés arithmétiques de rang rationnel deux, C. R. Acad. Sci. Paris 301 (1985), 369-373.

[BoT] R. Bott and L. Tu, Differential forms in algebraic topology, Springer-Verlag, Berlin and New York, 1982.

[C1] J. Cheeger, Hodge theory of complex cones, Analyse et Topologie sur les Espaces Singulièrs, Astérisque, vol. 101-102, Soc. Math. France, 1983, pp. 118-134.

[C2] _ On the Hodge theory of Riemannian pseudomanifolds, Proc. Sympos. Pure Math., vol. 36, Amer. Math. Soc., Providence, RI, 1980, pp. 91-146.

[C3] _ Spectral geometry of singular Riemannian spaces, J. Differential Geom. 18 (1983), 575-657.

[C4] _ On the spectral geometry of spaces with cone-like singularities, Proc. Nat. Acad. Sci. U.S.A. 76 (1979), 2103-2106.

[CGM] J. Cheeger, M. Goresky, and R. MacPherson, L ${ }^{2}$-cohomology and intersection homology of singular algebraic varieties, Seminar on Differential Geometry (S. T. Yau, ed.), Princeton Univ. Press, Princeton, NJ, 1982, pp. 303-340.

[GM1] M. Goresky and R. MacPherson, Intersection homology theory, Topology 19 (1980), 135162 .

[GM2] _ Intersection homology. II, Invent. Math. 71 (1983), 77-129.

[GrHa] P. Griffiths and J. Harris, Principles of algebraic geometry, Wiley, New York, 1978.

[H1] P. Haskell, Index theory on curves, Trans. Amer. Math. Soc. 288 (1985), 591-604.

[H2] _,$L^{2}$-Dolbeault complexes on singular curves and surfaces, Proc. Amer. Math. Soc. 107 (1989), 517-526.

[HsP] W. C. Hsiang and V. Pati, $L^{2}$-cohomology of normal algebraic surfaces I, Invent. Math. 81 (1985), 395-412.

[L] E. Looijenga, $L^{2}$-cohomology of locally symmetric varieties, Compositio Math. 67 (1988), 3-20.

[N1] M. Nagase, On the heat operators of normal singular algebraic surfaces, J. Differential Geom. 28 (1988), 37-57.

[N2] Pure Hodge structure of the harmonic $L^{2}$-forms on singular algebraic surfaces, Publ. Res. Inst. Math. Sci. 24 (1988), 1005-1023.

[N3] Remarks on the $L^{2}$-cohomology of singular algebraic surfaces, J. Math. Soc. Japan 41 (1989), 97-116.

[Pa] W. Pardon, The $L^{2}-\bar{\partial}$-cohomology of an algebraic surface, Topology 28 (1989), 171-195.

[S] M. Saito, Modules de Hodge polarisables, Publ. Res. Inst. Math. Sci. 24 (1988), 849-995.

[Sa1] L. Saper, $L_{2}$-cohomology and intersection homology of certain algebraic varieties with isolated singularities, Invent. Math. 82 (1985), 207-255.

[Sa2] _,$L_{2}$-cohomology of Kähler varieties with isolated singularities, J. Differential Geom. 36 (1992), 89-161.

[SaSt1] L. Saper and M. Stern, $L_{2}$-cohomology of arithmetic varieties, Proc. Nat. Acad. Sci. U.S.A. 84 (1987), 5516-5519.

[SaSt2] _ , L L-cohomology of arithmetic varieties, Ann. of Math. (2) 132 (1990), 1-69.

[Z1] S. Zucker, The Hodge structures on the intersection homology of varieties with isolated singularities, Duke Math. J. 55 (1987), 603-616.

[Z2] _,$L_{2}$-cohomology and intersection homology of locally symmetric varieties, II, Compositio Math. 59 (1986), 339-398.

[Z3] _ L L -cohomology of warped products and arithmetic groups, Invent. Math. 70 (1982), $169-218$.

Department of Mathematics, University of Colorado, Boulder, Colorado 80309

E-mail address: jfox@euclid.colorado.edu

Department of Mathematics, Virginia Polytechnic Institute and State University, Blacksburg, Virginia 24061

E-mail address: haskell@math.vt.edu 